\title{
Analysis of intestinal flora and inflammatory cytokine levels in children with non-infectious diarrhea
}

\author{
Lijia $\mathrm{Li}^{1 \#}$, Qingqing Yan $^{1 \#}, \mathrm{Na} \mathrm{Ma}^{1}$, Xiuling $\mathrm{Chen}^{2}$, Guiling $\mathrm{Li}^{1}, \mathrm{Min} \mathrm{Liu}^{2}$ \\ ${ }^{1}$ Department of Pediatrics, the First Affiliated Hospital of Hainan Medical University, Haikou, China; ${ }^{2}$ Department of Pediatrics, Central South \\ University Xiangya School of Medicine Affiliated Haikou Hospital, Haikou, China \\ Contributions: (I) Conception and design: L Li, Q Yan, M Liu; (II) Administrative support: N Ma, X Chen; (III) Provision of study materials or \\ patients: L Li, Q Yan, G Li; (IV) Collection and assembly of data: L Li, Q Yan, M Liu; (V) Data analysis and interpretation: L Li, Q Yan, M Liu; \\ (VI) Manuscript writing: All authors; (VII) Final approval of manuscript: All authors. \\ "These authors contributed equally to this work. \\ Correspondence to: Min Liu. Department of Pediatrics, Haikou Affiliated Hospital of Central South University Xiangya School of Medicine, Haikou \\ 570208, China. Email: chenyuxilm@163.com.
}

Background: Non-infectious diarrhea is a common symptom in infants and young children. We aimed to analyze the intestinal flora and serum inflammatory cytokine levels of children with non-infectious diarrhea.

Methods: Eighty-nine children with non-infectious diarrhea and 76 healthy children were enrolled from the First Affiliated Hospital of Hainan Medical University between February 2017 and June 2020. Fecal bacterial samples were collected in sterile containers. Following serial dilution, the bacterial samples were cultured in an aerobic medium to cultivate Escherichia coli (E. coli), Enterococci, Lactobacilli, and Bifidobacteria. The levels of inflammatory cytokines in the serum, including interleukin (IL)-2, IL-8, IL-10, and tumor necrosis factor-alpha (TNF- $\alpha$ ), were determined by enzyme-linked immunosorbent assay. Results between the groups were compared using the paired $t$-test. The chi-square test was employed to analyze categorical data, with analysis of variance used for multiple-group comparisons.

Results: No significant differences were observed between the diarrhea and control groups in terms of sex, age, or body mass index distribution. Compared to the control group, the diarrhea group had significantly elevated levels of E. coli and Enterococci but significantly decreased levels of Bifidobacteria and Lactobacilli. In terms of inflammatory cytokines, the levels of IL-2, IL-8, IL-10, and TNF- $\alpha$ were significantly higher in the diarrhea group than in the control group (all $\mathrm{P}<0.05$ ). In children with non-infectious diarrhea, the levels of IL-2, IL-8, IL-10, and TNF- $\alpha$ were positively correlated with the amount of $E$. coli ( $\mathrm{r}$ values of 0.412 , $0.381,0.479$, and 0.216, respectively) and Enterococci (r values of 0.257,0.336, 0.357, and 0.328). Further, the amount of Lactobacilli was positively correlated with IL-2 and IL-10 levels ( $\mathrm{r}$ values of 0.342 and 0.438 , respectively), and that of Bifidobacteria was negatively correlated with IL-2, IL-8, IL-10, and TNF- $\alpha$ levels ( $\mathrm{r}$ values of $-0.252,-0.336,-0.328$, and -0.293 , respectively). Finally, the level of Lactobacilli was also negatively correlated with IL- 8 and TNF- $\alpha$ levels ( $r$ values -0.301 and -0.464 , respectively; both $\mathrm{P}<0.05$ ).

Conclusions: The abundance and abnormality of E. coli, Enterococci, Lactobacilli, and Bifidobacteria in the intestinal flora of children with non-infectious diarrhea are associated with increased levels of IL-2, IL-8, IL-10, and TNF- $\alpha$.

Keywords: non-infectious diarrhea; intestinal flora; inflammatory cytokines.

Submitted Mar 10, 2021. Accepted for publication Apr 29, 2021.

doi: $10.21037 / \mathrm{tp}-21-168$

View this article at: http://dx.doi.org/10.21037/tp-21-168 


\section{Introduction}

Diarrhea is a major cause of infant and child morbidity and death, and poses a major threat to children, especially in developing countries (1). Various factors and pathogens can trigger the occurrence of diarrhea, the clinical features of which include the frequent passing of watery stools leading to dehydration and electrolyte imbalance (2). Depending on the disease course, diarrhea can be divided into 3 categories (acute, persistent, and chronic), and it can also be classified as infectious and non-infectious based on the etiology (3).

Due to their immature digestive system, low gastric acid level, and digestive enzyme activity, as well as their limited ability to adapt to changes in diet, infants and young children are susceptible to the occurrence of non-infectious diarrhea $(4,5)$. Moreover, as children have a fast growth rate and high nutritional requirements, their digestive organs are frequently in a tense state (6). For these reasons, children are prone to developing digestive disorders. Furthermore, digestive and absorption dysfunction in infants can increase the pressure in the intestinal cavity, inducing diarrhea (7). Chronic diarrhea in infants is often associated with congenital or genetic factors; for instance, allergic diarrhea is caused by allergies to a certain food ingredient, and malabsorption diarrhea is caused by lack of a particular enzyme (8).

The intestinal flora and inflammatory cytokines in the serum have been shown to play an important role in the pathogenesis of diarrhea (9). Currently, the clinical objective of treatment for non-infectious diarrhea is symptomatic relief (10). After a clinical diagnosis of non-infectious diarrhea, the doctor may request that a stool sample from the patient undergo microscopic examination to detect bacteria, parasites, or other microorganisms. Patients with no evidence of infectiousness after antigen testing and microscopic examination are generally diagnosed with non-infectious diarrhea (11). In a standard laboratory, the diagnosis of diarrhea relies on the use of a microscope, antigen detection, and culture of relevant bacteria, which can be both time-consuming and labor-intensive, and is typically associated with low detection efficiency.

According to the genetic basis and other pathophysiological characteristics of non-infectious diarrhea, stool analysis and serum testing can aid doctors in the evaluation and treatment management of children with diarrhea. Therefore, in this study, we aimed to evaluate the changes in the intestinal flora and serum inflammatory cytokine levels of children with non-infectious diarrhea, with a view to improving the diagnostic and treatment efficiency for such patients.
We present the following article in accordance with the STROBE reporting checklist (available at http://dx.doi. org/10.21037/tp-21-168).

\section{Methods}

\section{Research participants}

This study enrolled 89 children with non-infectious diarrhea and 76 healthy children in the First Affiliated Hospital of Hainan Medical University between February 2017 and June 2020. The 86 boys and 79 girls participating in the study ranged in age from 4 months to 7 years old. The inclusion criteria included: (I) a diagnosis of non-infectious diarrhea; (II) bowel movements $>5 /$ day; (III) a change in stool characteristics determined based on the child's medical history, clinical manifestations, and related factors. The exclusion criteria were: (I) a diagnosis of infectious diarrhea; (II) severe vomiting, diarrhea, and dehydration; (III) severe organic diseases or other infectious diseases. All procedures performed in this study involving human participants were in accordance with the Declaration of Helsinki (as revised in 2013). The study was approved by the Ethics Committee of the First Affiliated Hospital of Hainan Medical University (NO.: 2017021) and informed consent was taken from all the patients.

\section{Data collection}

Fecal bacterial samples from the children were collected in sterile containers. Following serial dilution, the samples were cultured in an aerobic medium to cultivate Escherichia coli (E. coli) and Enterococci. An anaerobic medium was also used to cultivate Lactobacilli and Bifidobacteria. After incubation for 3 days at $37^{\circ} \mathrm{C}$, the colonies were counted and recorded as the logarithm of colony-forming units (CFUs)/gram of wet fecal weight. The levels of the inflammatory cytokines interleukin (IL)-2, IL-8, IL-10, and tumor necrosis factoralpha $(\mathrm{TNF}-\alpha)$ in the serum were determined by enzymelinked immunosorbent assay (ELISA) (Abcam, UK).

\section{Statistical analysis}

SPSS 17.0 software (SPSS Corporation, Chicago, Illinois, USA) was used for data analysis. All measurements were represented by mean \pm standard deviation. Results between the groups were compared using the paired $t$-test. The chisquare test was employed to analyze categorical data, with analysis of variance used for multiple-group comparisons. 
Table 1 Comparison of basic data between the diarrhea group and the control group

\begin{tabular}{lcccc}
\hline Groups & Cases & Male, $\mathrm{n}(\%)$ & Age (year) & $\mathrm{BMl}\left(\mathrm{kg} / \mathrm{m}^{2}\right)$ \\
\hline Diarrhea group & 89 & $49(55.1)$ & $3.47 \pm 1.89$ & $24.15 \pm 1.12$ \\
Control group & 76 & $37(48.7)$ & $3.96 \pm 1.81$ & $26.28 \pm 1.28$ \\
P value & & 0.078 & 0.081 & 0.062 \\
\hline
\end{tabular}

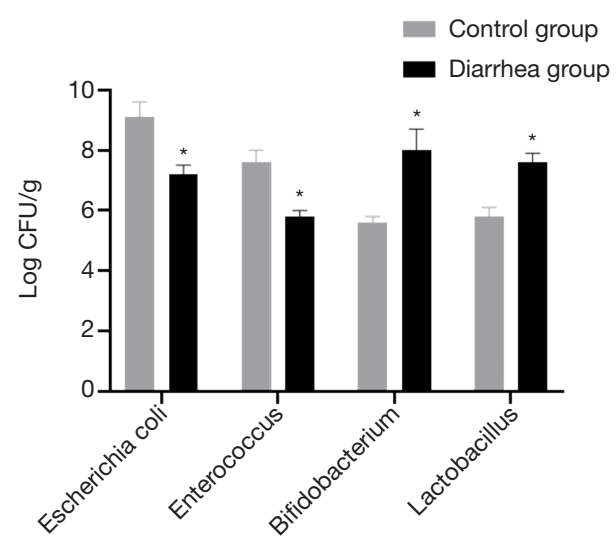

Figure 1 Comparison of the intestinal flora between the diarrhea group and the control group $\left({ }^{*} \mathrm{P}<0.05\right)$.

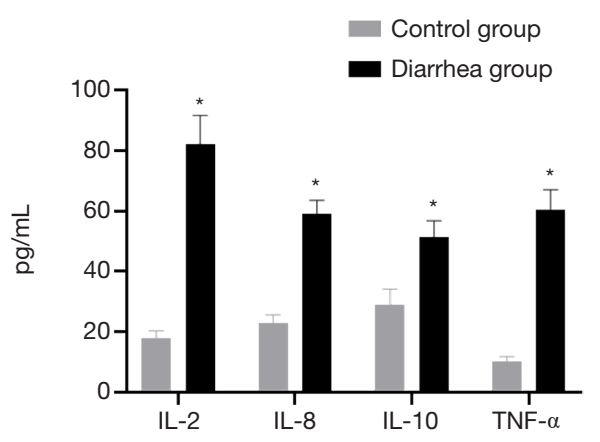

Figure 2 Comparison of serum inflammatory cytokine levels between the diarrhea group and the control group $\left({ }^{*} \mathrm{P}<0.05\right)$.

$\mathrm{P}<0.05$ showed a statistically significant difference.

\section{Results}

\section{Comparison of basic data between the diarrbea group and the control group}

The average age of the study participants was $3.47 \pm 1.89$ years old and the average BMI was $24.15 \pm 1.12 \mathrm{~kg} / \mathrm{m}^{2}$. As shown in Table 1, no significant difference existed between the groups in terms of sex, age, or BMI (all $\mathrm{P}>0.05$ ).

\section{Comparison of the intestinal flora between the diarrbea group and the control group}

Examination of the intestinal flora samples revealed $7.2 \pm 0.3$ and $9.1 \pm 0.5(\log \mathrm{CFU} / \mathrm{g})$ of E. coli, $5.8 \pm 0.2$ and

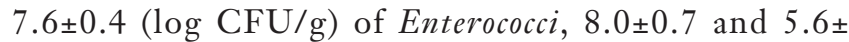
$0.2(\log$ CFU/g) of Bifidobacteria, and 7.6 \pm 0.3 and $5.8 \pm$ $0.3(\log$ CFU/g) of Lactobacilli in the diarrhea group and the control group, respectively. Compared to the control group, the diarrhea group had significantly lower levels of E. coli and Enterococci, but significantly higher levels of Bifidobacteria and Lactobacilli (all $\mathrm{P}<0.05$ ), as shown in Figure 1.

\section{Comparison of serum inflammatory cytokine levels between the diarrbea and control groups}

The levels of inflammatory cytokines in the participants' serum were also analyzed. In the diarrhea group, the serum levels of IL-2, IL-8, IL-10, and TNF- $\alpha$ were $82.1 \pm 9.4$, $59.1 \pm 4.4,51.3 \pm 5.4$, and $60.5 \pm 6.5 \mathrm{pg} / \mathrm{mL}$, respectively. In the control group, the serum levels of IL-2, IL-8, IL-10, and TNF- $\alpha$ were $17.8 \pm 2.6,23.1 \pm 2.6,29.1 \pm 5.1$, and $10.1 \pm$ $1.8 \mathrm{pg} / \mathrm{mL}$, respectively. As visualized in Figure 2, the diarrhea group had significantly higher levels of IL-2, IL-8, IL-10, and TNF- $\alpha$ than the control group (all $\mathrm{P}<0.05$ ).

\section{Relationship between the abundance of different bacteria and serum inflammatory cytokine levels in children with non-infectious diarrbea}

Analysis of the intestinal flora and serum inflammatory cytokine levels of children with non-infectious diarrhea revealed that the levels of IL-2, IL-8, IL-10, and TNF- $\alpha$ were significantly positively correlated with the abundance of $E$. coli (r value: $0.412,0.381,0.479$, and 0.216, respectively) and Enterococci ( $\mathrm{r}$ value: $0.257,0.336$, 0.357 , and 0.328 , respectively). Furthermore, significant positive correlations were found between the abundance 
Table 2 Correlation between the abundance of different bacteria and serum inflammatory cytokine levels in children with non-infectious diarrhea

\begin{tabular}{|c|c|c|c|c|c|c|c|c|}
\hline Bacterial & \multicolumn{2}{|c|}{ IL-2 } & \multicolumn{2}{|c|}{ IL-8 } & \multicolumn{2}{|c|}{ IL-10 } & \multicolumn{2}{|c|}{ TNF- $\alpha$} \\
\hline E. coli & 0.412 & 0.007 & 0.381 & 0.03 & 0.479 & 0.001 & 0.216 & 0.002 \\
\hline Enterococci & 0.257 & 0.006 & 0.336 & 0.008 & 0.357 & 0.002 & 0.328 & 0.004 \\
\hline Bifidobacteria & -0.252 & 0.01 & -0.336 & 0.002 & -0.328 & 0.009 & -0.293 & 0.006 \\
\hline
\end{tabular}

of Lactobacilli and the levels of IL-2 and IL-10 (r value: 0.342 and 0.438 , respectively), whereas significant negative correlations existed between the abundance of Bifidobacteria and the levels of IL-2, IL-8, IL-10, and TNF- $\alpha$ (r value: $-0.252,-0.336,-0.328$ and -0.293 , respectively). Finally, the abundance of Lactobacilli was also negatively correlated with the levels of IL- 8 and TNF- $\alpha$ ( $r$ value: -0.301 and -0.464 , respectively) (all $\mathrm{P}<0.05$ ), as shown in Table 2.

\section{Discussion}

It has been estimated that the human digestive tract contains hundreds of bacteria and that there are thousands of microorganisms in every gram of feces, including anaerobic, facultative anaerobes, and aerobic bacteria (12). The human intestinal flora typically forms between 1 and 2 years after birth, and its normal development is essential for intestinal function and human health (13). Imbalance of the intestinal flora can stimulate the release of inflammatory cytokine, which is harmful (14). Research has found that intestinal diseases are tightly associated with intestinal microecology, and increasing attention has been paid to the effects of intestinal flora imbalance on the progression of intestinal diseases (15). Most microorganisms in the small intestine are gram-positive cocci and rod-shaped bacteria, which are extremely important for intestinal functional maintenance; however, their excessive growth can lead to intestinal problems (16). Anaerobic bacteria, including bacillus and bifidobacterium (17), are major players in the human large intestine. This study has demonstrated that the E. coli and Enterococci in children with non-infectious diarrhea were significantly increased, while those of Bifidobacteria and Lactobacilli were significantly reduced. Therefore, children with non-infectious diarrhea can develop intestinal flora imbalance, with a decreased level of probiotics and an increased level of harmful bacteria.

Reports have suggested that the effectiveness of interventional treatment for intestinal diseases can be enhanced by regulating the intestinal flora and serum inflammatory cytokine levels $(18,19)$. Pro-inflammatory and anti-inflammatory cytokines both play important roles in the pathogenesis of intestinal diseases. Studies have shown increased levels of IL-2, IL-6, and IL-8 in mice with small bowel diseases (20). Furthermore, after treatment with probiotics, the expression level of the above-mentioned inflammatory cytokine (IL-2, IL-6, and IL-8) was downregulated, and the inflammation of the intestinal mucosa was also reduced (21). A large cross-sectional study in the United Kingdom showed that different expression levels of serum inflammatory cytokines can distinguish between infectious and non-infectious diarrhea (22). IL-2, IL-8, TNF- $\alpha$, and other inflammatory cytokines are considered to be the key proinflammatory factors participating in the mediation of the occurrence and development of inflammation in the body (23). Changes in the expression levels of IL-8 at different stages of diarrhea can also be useful for determining the prognosis (24-26). In this study, the levels of IL-2, IL-8, IL-10, and TNF- $\alpha$ in the serum of children with non-infectious diarrhea were significantly higher than those in the control group, suggesting that the abundance of these inflammatory cytokines might be associated with the progression of non-infectious diarrhea.

The tight relationship between the intestinal flora and inflammatory cytokines has been extensively studied, and it is now understood that intestinal diseases can be treated more effectively through alterations to the intestinal flora. One study analyzed the correlation between inflammation and the intestinal flora of patients with Crohn's disease, and found that the levels of inflammatory cytokines are closely related to the intestinal flora (27). The present study showed that E. coli and Enterococci are positively correlated with the levels of serum IL-2, IL-8, IL-10, and TNF- $\alpha$ in children with non-infectious diarrhea, while Bifidobacteria and Lactobacilli are negatively associated with the levels of 
these cytokines. The results therefore suggest that altering the intestinal flora may influence the secretion of certain inflammatory cytokines in the body.

This study has a number of limitations. Firstly, we did not evaluate the interactions of these three bacterial $(E$. coli, Enterococci, Bifidobacteria, and Lactobacilli) abundances in healthy individuals. Further, the method of using simple agar-cultured microbiota in this study also needs improvement. Our preliminary findings have shown that there is a correlation between intestinal bacteria and inflammatory cytokines, which is consistent with the results of previous studies on osmotic diarrhea in which alterations in cytokine levels were found to affect the long-lasting IgG antibacterial response (28). Therefore, multiple factors may have the potential to serve as a diagnostic biomarker of non-infectious diarrhea. However, the levels of cytokines detected in the serum may also reflect inflammation beyond the local area. Therefore, we believe that a large number of bacteria may induce the release of systemic proinflammatory cytokines, and the underlying mechanism remains to be explored in future investigations.

In summary, we have demonstrated that changes and abnormalities of E. coli, Enterococci, Lactobacilli, and Bifidobacteria in the intestinal flora of children with noninfectious diarrhea are related to increased levels of IL-2, IL-8, IL-10, and TNF- $\alpha$.

\section{Acknowledgments}

Funding: None.

\section{Footnote}

Reporting Checklist: The authors have completed the STROBE reporting checklist. Available at http://dx.doi. org/10.21037/tp-21-168

Data Sharing Statement: Available at http://dx.doi. org/10.21037/tp-21-168

Conflicts of Interest: All authors have completed the ICMJE uniform disclosure form (available at http://dx.doi. org/10.21037/tp-21-168). The authors have no conflicts of interest to declare.

Ethical Statement: The authors are accountable for all aspects of the work in ensuring that questions related to the accuracy or integrity of any part of the work are appropriately investigated and resolved. All procedures performed in this study involving human participants were in accordance with the Declaration of Helsinki (as revised in 2013). The study was approved by the Ethics Committee of the First Affiliated Hospital of Hainan Medical University (NO.: 2017021) and informed consent was taken from all the patients.

Open Access Statement: This is an Open Access article distributed in accordance with the Creative Commons Attribution-NonCommercial-NoDerivs 4.0 International License (CC BY-NC-ND 4.0), which permits the noncommercial replication and distribution of the article with the strict proviso that no changes or edits are made and the original work is properly cited (including links to both the formal publication through the relevant DOI and the license). See: https://creativecommons.org/licenses/by-nc-nd/4.0/.

\section{References}

1. Conlon MA, Bird AR. The impact of diet and lifestyle on gut microbiota and human health. Nutrients 2014;7:17-44.

2. Singh RK, Chang HW, Yan D, et al. Influence of diet on the gut microbiome and implications for human health. J Transl Med 2017;15:73.

3. Brennan CA, Garrett WS. Gut microbiota, inflammation, and colorectal cancer. Annu Rev Microbiol 2016;70:395-411.

4. Valdes AM, Walter J, Segal E, et al. Role of the gut microbiota in nutrition and health. BMJ 2018;361:k2179.

5. Qin J, Li R, Raes J, et al. A human gut microbial gene catalogue established by metagenomic sequencing. Nature 2010;464:59-65.

6. Almeida A, Mitchell AL, Boland M, et al. A new genomic blueprint of the human gut microbiota. Nature. 2019;568:499-504.

7. Rothschild D, Weissbrod O, Barkan E, et al. Environment dominates over host genetics in shaping human gut microbiota. Nature 2018;555:210-5.

8. Noble RE. Waist-to-hip ratio versus BMI as predictors of cardiac risk in obese adult women. West J Med 2001;174:240-1.

9. Hamer M, O'Donovan G, Stensel D, et al. NormalWeight central obesity and risk for mortality. Ann Intern Med 2017;166:917-8.

10. Gentile CL, Weir TL. The gut microbiota at the intersection of diet and human health. Science 2018;362:776-80. 
11. Lauderdale DS, Rathouz PJ. Body mass index in a US national sample of Asian Americans: Effects of nativity, years since immigration and socioeconomic status. Int $\mathrm{J}$ Obes Relat Metab Disord 2000;24:1188-94.

12. Vangay P, Johnson AJ, Ward TL, et al. US immigration westernizes the human gut microbiome. Cell 2018;175:962-972.e10.

13. Arumugam M, Raes J, Pelletier E, et al. Enterotypes of the human gut microbiome. Nature 2011;473:174-80.

14. Wu GD, Chen J, Hoffmann C, et al. Linking long-term dietary patterns with gut microbial enterotypes. Science 2011;334:105-8.

15. Rajilić-Stojanović M, Jonkers DM, Salonen A, et al. Intestinal microbiota and diet in IBS: Causes, consequences, or epiphenomena? Am J Gastroenterol 2015;110:278-87.

16. Hall AB, Yassour M, Sauk J, et al. A novel Ruminococcus gnavus clade enriched in inflammatory bowel disease patients. Genome Med 2017;9:103.

17. Henke MT, Kenny DJ, Cassilly CD, et al. Ruminococcus gnavus, a member of the human gut microbiome associated with Crohn's disease, produces an inflammatory polysaccharide. Proc Natl Acad Sci U S A 2019;116:12672-7.

18. Kim ER, Chang DK. Colorectal cancer in inflammatory bowel disease: The risk, pathogenesis, prevention and diagnosis. World J Gastroenterol 2014;20:9872-81.

19. Jandhyala SM, Talukdar R, Subramanyam C, et al. Role of the normal gut microbiota. World J Gastroenterol 2015;21:8787-803.

20. Winter SE, Baumler AJ. Dysbiosis in the inflamed intestine: Chance favors the prepared microbe. Gut

Cite this article as: Li L, Yan Q, Ma N, Chen X, Li G, Liu M. Analysis of intestinal flora and inflammatory cytokine levels in children with non-infectious diarrhea. Transl Pediatr 2021;10(5):1340-1345. doi: 10.21037/tp-21-168
Microbes 2014;5:71-3.

21. Martinez-Medina M, Garcia-Gil LJ. Escherichia coli in chronic inflammatory bowel diseases: An update on adherent invasive Escherichia coli pathogenicity. World J Gastrointest Pathophysiol 2014;5:213-27.

22. Lewis JD, Chen EZ, Baldassano RN, et al. Inflammation, antibiotics, and diet as environmental stressors of the gut microbiome in pediatric Crohn's disease. Cell Host Microbe 2015;18:489-500.

23. Margolis DJ, Fanelli M, Hoffstad O, et al. Potential association between the oral tetracycline class of antimicrobials used to treat acne and inflammatory bowel disease. Am. J. Gastroenterol. 2010;105:2610-6.

24. Lloyd DA, Powell-Tuck J. Artificial nutrition: Principles and practice of enteral feeding. Clin. Colon Rectal Surg 2004;17:107-18.

25. Llewellyn SR, Britton GJ, Contijoch EJ, et al. Interactions between diet and the intestinal microbiota alter intestinal permeability and colitis severity in mice. Gastroenterology 2018;154:1037-1046.e2.

26. Chiba M, Abe T, Tsuda H, et al. Lifestyle-related disease in Crohn's disease: Relapse prevention by a semivegetarian diet. World J Gastroenterol 2010;16:2484-95.

27. Lewis JD, Abreu MT. Diet as a trigger or therapy for inflammatory bowel diseases. Gastroenterology 2017;152:398-414.e6.

28. Roy CC, Kien CL, Bouthillier L, et al. Short-chain fatty acids: Ready for prime time? Nutr Clin Pract 2006;21:351-66.

(English Language Editor: J. Jones) 